\title{
The effects of category use on learned categories
}

\author{
BRIAN H. ROSS \\ University of Illinois at Urbana-Champaign, Urbana, Illinois
}

\begin{abstract}
Frequently, people learn to classify instances of a concept and later learn additional information about the concept. What is the effect of this later learning on the original classification? In five experiments, this issue was investigated with a common classification paradigm in which symptom sets were classified into disease categories. After learning to classify these sets, the subjects learned to use the category to decide what treatment should be given for a symptom set. The symptoms that were important for the treatments were later classified by disease more accurately and were generated earlier from the disease name. However, this effect occurred only if the category representation was activated during the learning of the treatments. Thus, later learning about a particular use of the concept can sometimes affect the original classification.
\end{abstract}

As we learn about the world, we acquire new concepts and augment the conceptual representations we already have. A central issue in concept learning is how conceptual representations change with additional experience. The goal of this paper is to address one aspect of such conceptual change: the effect of later learning on an initially learned classification.

Research on category learning has focused primarily on classification-on how people learn to assign new instances to categories (e.g., Kruschke, 1992; Lamberts, 1994; Medin \& Schaffer, 1978; Nosofsky, 1988; Rosch, 1978; see Medin \& Smith, 1984; Ross \& Spalding, 1994; Smith \& Medin, 1981, for reviews). However, in order for categories to be useful, we must do more than simply learn to classify. An important aspect of categories is that they provide access to relevant knowledge for a variety of tasks. This access leads to additional information which can be useful for accomplishing category-based goals such as inference, prediction, explanation, and problem solving. Thus, learning new categories involves both learning to classify and learning to make use of the category. We must ask the question, How is the original classification affected by this additional learning?

\section{Category Use}

In much of the previous work on using categories, researchers have examined how people use established cat-

This work was partially supported by NSF Grant SBR 97-20304. Research for this paper was conducted at the Beckman Institute for Advanced Science and Technology. I thank Gregory Murphy for a number of discussions and comments on the manuscript, and Lawrence Barsalou, Gary Dell, Susan Gelman, Arthur Markman, Douglas Medin, Colleen Seifert, and Edward Smith for discussions of this research. 1 also thank Amy Anderson, Corinna Crawford, Adam Joncinch, Catherine Kennedy, Amanda Lorenz, Amanda Schulze, and Holly Trytten for their help in conducting the experiments. Correspondence may be addressed to B. H. Ross, Beckman Institute, University of Illinois, 405 N. Mathews Ave., Urbana, IL 61801 (e-mail: bross@s.psych.uiuc. edu). egories to make inductions or predict features when the category is known (e.g., Gelman \& E. M. Markman, 1986; Heit, 1992; Osherson, Smith, Wilkie, Lopez, \& Shafir, 1990) or is uncertain (e.g., Anderson, 1991; Malt, Ross, \& Murphy, 1995; Murphy \& Ross, 1994; Ross \& Murphy, 1996). However, they have not examined how additional information that is learned about the categories might affect the classification.

Some results suggest that extensive experience in using categories may influence later classifications. As people gain more experience in a domain, for example, they may classify objects at a more specific level (see, e.g., Gibson, 1969; Tanaka \& Taylor, 1991). Medin and his colleagues (Lopez, Atran, Coley, Medin, \& Smith, 1997; Medin, Lynch, Coley, \& Atran, 1997) have found that experts' classification sortings are influenced by the categories' use. For example, landscapers' sortings of trees were greatly influenced by whether the trees were ornamental or provided shade (see also Boster \& Johnson, 1989; Malt, 1995). In problem-solving domains, experts rely on different, more abstract features of problems for classifying problems than do novices (e.g., Chi, Feltovich, \& Glaser, 1981). However, the studies on expertise do not control the type of domain experience, so the findings do not show that learning to use already learned categories will influence classification.

When classifications are not provided during learning, experience with category members in a nonclassification task may affect the categories formed as well as the later classifications (e.g., Billman \& Heit, 1988; Billman \& Knutson, 1996; Lassaline \& Murphy, 1996; Ward \& Becker, 1992). Barsalou (1983, 1985, 1991) has shown that for particular goals, people can form new ad hoc categories that affect the classifications of items. Yamauchi and A. B. Markman (1998; A. B. Markman, Yamauchi, \& Makin, 1997) have included a more direct comparison of classification and category use and have suggested that classification learning leads to the learning of distinguishing features, whereas a feature prediction task leads 
to learning more about the commonalities among exemplars within a category.

The work closest to the present investigation is that of Ross (1996b, 1997; see 1996a for an overview), which demonstrates that how people interact with category items or use them to make further predictions can affect classification. Ross (1997), using a task like the one in the present experiments, had subjects learn to diagnose "patients" (who were really sets of symptoms) as having fictitious diseases and then to decide, for each patient, what drug treatment should be given (a type of feature prediction). The symptoms that were predictive of the treatments came to be viewed as more central to the disease than other symptoms that were not predictive of the treatments, though, in fact, both types of symptoms were equally predictive of the disease and were presented equally often. Related effects were found with two different problem-solving uses of the categories. Thus, when people concurrently learn to classify and to use categories, the category use may affect the classification. The features relevant to the use may come to be viewed as being more relevant for the classification. Ross called this the category use effect. It suggests that how we classify may not just be a function of classification knowledge (and feedback about the classification), it may involve nonclassification knowledge about the concept.

\section{Postclassification}

In previous work on category use effects (e.g., Ross, 1997), the focus has been on how the classification is affected, but the classification and use have been learned interleaved (i.e., each item was classified and then this classified item was used to make a further prediction). In the present article, the work on category use is extended to examine how an already learned classification is affected by later learning. Subjects first learned a classification ("Which disease does this patient have?"). Following this learning, the subjects learned to predict an additional feature for the item ("Which treatment should this patient get?"). The question of interest was how the subjects' original classification might change as a function of this additional learning. I call this learning paradigm, in which additional information is learned after the original classification, a postclassification paradigm. Such a learning situation is common to many category uses. For example, we are often familiar with the basic categories in a domain before we learn to use these categories to accomplish some goal. In addition, the separation of the two learning tasks provides two advantages. First, it is possible that the effect occurs only in the interleaved paradigm because the subjects are trying to learn the features that will allow accurate responding in both tasks. The postclassification paradigm provides a check on this possibility. Second, the separation permits a better analysis of the conditions necessary for the effect to occur.

In parallel to this investigation with a prediction task, I have also examined postclassification effects in a problem- solving paradigm (Ross, 1999). In this problem-solving situation, the use of the category (to apply a formula to the numbers in an item) does have an effect on the category representation, and thus on later classification. However, that work differs from the present investigation in three important ways. First, in Ross (1999), the features learned from the use were ones that could not have been learned in the original classification, and the subjects knew to focus on the other features during the original classification. Second, these features learned from the use were not simple, observable features, but were derived from the problem-solving use of the category (e.g., learning that the product of the numbers in two positions was 12 for all of the items in a particular category). Third, the tests showed that these new features were used for classification when the originally learned classification features were not available. Thus, the new features had been added to the category representation, but the study was not designed for the purpose of examining whether this additional learning would have any effect on the features originally learned for classification. These are significant differences that largely arise from some of the differences between problem-solving and feature prediction category uses. The problem-solving studies in Ross (1999) were designed to examine ideas from the expertise literature, in which extensive experience leads to a change in the features involved in the items' representations (e.g., Chi et al., 1981). The present work is more similar to the standard classification learning paradigm in which subjects know what all the features are and have to decide how to combine them to make classification decisions. Although the derivation of new features is an interesting new area of research in category learning (e.g., Schyns, Goldstone, \& Thibaut, 1998; Schyns \& Rodet, 1997), it is important to consider how a postclassification learning of category use might affect the original representation in the standard task (and common situation) when all the features are known.

Why might the additional learning affect classification performance even when the classification has already been learned? This question will be addressed further in the General Discussion, but a brief answer may be given here. As one learns more about a concept, the representation is changed. This additional learning might lead to a change in the weighting of features, new features being learned, new relations among features being encoded, and so forth. When this representation is used to classify a new item, the changes in the representation might affect the classification. For example, suppose some feature that was largely ignored in the original classification is learned as being important to a use of the category. When one later has to classify an item into the original categories and one accesses the conceptual representations to decide the correct category, this feature may prove to be more influential than it was before the additional learning occurred. The point here is that the classification knowledge may not be isolated from whatever else we know about the concept, and so, as we 
learn more about a concept, the classification can be affected.

In summary, in the experiments reported in this paper, the effects of later learning on an already learned classification were examined. This is important for our understanding of category representation and category use in the postclassification paradigm.

\section{Subclassification}

Before presenting the experiments, I will mention one further theoretical issue. Although the motivation for these experiments has been given as an examination of postclassification category use, the experiments can also be viewed as an examination of how people learn further distinctions within a category - that is, a type of subclassification. For example, for the task to be used in the experiments in this paper, if a subject first decides what disease a patient has (classification) and then decides which of two disease-appropriate treatments the patient should get (prediction), the second decision can also be viewed as a division of the patients with that disease into groups who get one type of treatment and groups who get the other treatment. Anderson (1991) argues that all classifications can be viewed as feature prediction tasks. Although there may be important psychological differences between predicting category labels and other features (e.g., E. M. Markman, 1989; Murphy, 1993; Yamauchi \& A. B. Markman, 1998), in some cases it seems that learners may view a prediction as a subclassification.

Many concepts are part of a hierarchy. We often know much about the superordinates of concepts (e.g., an apple is a fruit; a dog is a mammal) and subordinates (e.g., there are many types of apples, such as Delicious, Macintosh, etc.; there are many types of dogs, such as collies, terriers, etc.). These hierarchies are crucial for being able to make inferences (e.g., does this new type of dog have a heart?). Although many types of concepts can be viewed as part of a hierarchy, most of the research on classification learning has considered learning at a single level, without regard to the superordinates or subordinates of the concept. There have been some exceptions, with research on basic levels (e.g., Johnson \& Mervis, 1997; Tanaka \& Taylor, 1991), expertise (Chi et al., 1981), and the learning of subcategories in children (e.g., Waxman, Lynch, Casey, \& Baer, 1997). However, little work has been done to examine how the original classification is affected by this later learning of subcategories. Although in the experiments in this article I did not examine whether subjects represented these treatments as subcategories or features, it is useful to consider for future research that some studies with feature predictions may also be viewed as an examination of how learning subcategories affects the original classification.

\section{The Present Experiments}

In the five experiments reported here, category learning consisted of both learning to classify and then learn- ing to make a feature prediction or subclassification. Each experiment began with the usual classification learning paradigm: An item was presented, the subject classified the item into one of the experimenter-defined categories, and feedback was given. After the subjects reached a learning criterion on their classification, the additional learning of the category use was introduced. For this part of the learning, on each trial the subject had to make some further response about the item, and feedback was then given. The experiments were designed with the purpose of examining how this later learning would affect the category representation, including knowledge used to make subsequent classifications. This research addresses two questions: First, is there a postclassification effect when the additional learning is a feature prediction? Second, if so, what are the conditions under which it occurs? The answers to the latter question will provide additional constraints on how such an effect might be incorporated into a model of concept acquisition.

Experiment 1 provided an examination of the basic effect - does the additional learning following classification learning affect subsequent classifications? Experiment 2 extended this effect to a different category-related dependent measure and begins to examine the conditions necessary for this effect to occur. In Experiments 3-5, these conditions were examined further.

\section{EXPERIMENT 1}

In the first experiment, subjects learned to classify to a criterion, and only then was the use of the category to make further predictions introduced. The question was whether or not using the categories to learn to make further decisions might influence later classifications, even if a particular classification had already been learned. The materials and general procedure in this experiment were similar to those in Ross (1997, Experiment 1), which showed a strong influence of the use on classification when the learning of the classification and use were interleaved. Would a similar effect be found when subjects learned the classification first?

The items listed in this experiment were fictitious "patients," presented as sets of symptoms to be classified into fictitious disease categories on the basis of their symptoms (a common task for classification learning; see, e.g., Medin \& Edelson, 1988). After the subjects learned to classify the patients into disease categories, they continued classifying, but they also had to decide which treatment each classified patient should receive. Of the symptoms that were predictive of the disease, some were predictive of the treatment as well (called relevantuse symptoms), and some were not (called irrelevant-use symptoms). The tests of most interest were later disease classifications. If the treatment decisions that were learned after classification did not affect classification knowledge, there should be no differences between the relevant-use and irrelevant-use symptoms in later 
classifications. If, however, the relevant-use symptoms also came to be viewed as more central to the disease, these symptoms should be classified better than the irrelevant-use symptoms.

\section{Method}

Subjects. The subjects were 20 University of Illinois undergraduates who participated for pay. The sessions lasted $30-50 \mathrm{~min}$.

Materials. The materials, which were the same as those used in Experiment 1 of Ross (1997; loosely adapted from Medin \& Edelson, 1988), consisted of diseases, drug treatments, and symptoms. There were 2 fictitious diseases (buragamo and terrigitis), 4 fictitious drug treatments (lamohillin and pexlophene for buragamo; galudane and veptendrine for terrigitis), and 12 symptoms (fever, runny nose, dizziness, abdominal pain for buragamo; cough, nausea, sore muscles, earache for terrigitis; and skin rash, numb fingers, swollen tongue, and inflamed knee as nonpredictive symptoms).

All four symptoms for each disease were perfectly predictive of the disease. That is, whenever any of these symptoms was present the disease occurred. Of these symptoms, the two relevant-use symptoms were also perfectly predictive of a treatment. For example, if cough and nausea were the relevant-use symptoms for terrigitis, any patient with a cough would have the disease terrigitis and be treated with galudane, whereas any patient with nausea would have the disease terrigitis and be treated with veptendrine. The other two symptoms for a disease were irrelevant-use symptoms and occurred half the time with each of the disease treatments. To continue the example, any patient with sore muscles would also have the disease terrigitis but half the time would be treated with galudane (because the patient also had a cough) and half the time would be treated with veptendrine (because the patient also had nausea).

Each "patient" consisted of three symptoms: two that were from one of the diseases (a relevant-use symptom and an irrelevant-use symptom) and one symptom from the four nonpredictive symptoms. Sixteen patients were constructed in this way, with 8 from each disease category and 4 from each treatment. The four nonpredictive symptoms occurred once with each treatment (so twice with each disease). Thus, 1 patient might have a cough, sore muscles, and numb fingers. This patient would have terrigitis (as indicated by both of the first two symptoms) and would be treated with galudane (as indicated by the relevant-use symptom, cough). A partial design with sample items is given in Table 1.

The symptoms for a patient were typed onto a $3 \times 5$ in. $(7.6 \times$ $12.7 \mathrm{~cm}$ ) index card, and three cards were made for each patient (counterbalancing the order of symptoms). Each study block consisted of one presentation of each of the 16 patients. To ensure that any choice differences were not due to particular symptoms, the relevant-use and irrelevant-use symptoms were counterbalanced over subjects. The two types of symptoms were switched, and another set of cards was made for half the subjects.

The test materials consisted of single-symptom tests and doublesymptom tests. For the single-symptom tests, each of the 12 symp- toms was presented individually for the subject to classify by disease. For the 16 double-symptom tests, 2 symptoms were presented to the subject. For 8 of the tests, a relevant-use symptom for one disease was paired with an irrelevant-use symptom for the other disease. The other 8 double-symptom tests consisted of 2 symptoms for the same disease: both irrelevant-use symptoms (i.e., 0 relevantuse symptoms), 1 relevant-use symptom and 1 irrelevant-use symptom, or both relevant-use symptoms. The latter 8 tests were fillers, so that not all of the tests were conflicts between the two diseases.

Procedure. The learning part of the experiment consisted of a classification-only phase and a classification-use phase. For the classification-only phase, the subjects were told that they would be learning about some disease categories by diagnosing patients who had sets of different symptoms. They were given a sheet with the two disease names, which remained visible throughout this phase of the experiment. The instructions stressed that the subjects should try not to use prior medical knowledge and that the same symptom might occur with different diseases. On each study trial, the subject received a patient card with three symptoms and responded with one of the diseases. The subject received feedback and was allowed to study the card for as long as he/she wanted. Each block consisted of one presentation of each of the 16 patients, who were presented in a random order. The first three study blocks had different orders of symptoms for each patient, which were later reused in other blocks. The subject continued to respond until correct on at least 14 of the 16 items in a block or until they completed six blocks.

After the classification learning, subjects proceeded to the classification-use phase. They were told that after classifying each patient, they would have to decide which drug treatment should be given. A sheet with the two diseases and the two drugs for each disease was visible throughout this phase of the experiment. The same patients were used as in the classification-only phase. On each trial, the subject responded with a disease, received feedback, made a decision about the drug treatment (from the two possibilities for that disease), received feedback again, and then was allowed to study the card for as long as was necessary. The subjects continued this procedure until they had responded correctly at least 14 out of 16 times for both diseases and treatments, or until they had completed six blocks. (Note that this classification-use phase was identical to the full paradigm of interleaved learning in Ross, 1997, Experiment 1.)

Following the study blocks, two types of tests were given: singlesymptom tests followed by double-symptom tests. For the singlesymptom tests, each of the 12 symptoms was presented individually, and the subject responded with the disease that he/she thought most likely for a patient with the symptom, as well as a confidence rating (from a low of 1 to a high of 7). For the 16 double-symptom tests, two symptoms were presented, and the subject again responded with a disease name and confidence rating. Following these tests, the subjects were debriefed, and any questions that they had were answered.

Design. The main manipulation of relevant-use symptoms versus irrelevant-use symptoms for a disease was within subjects. The only between-subjects manipulation was the counterbalancing of

Table 1

Partial Design of Experiment 1: Sample Study Materials for Disease Terrigitis

\begin{tabular}{lllll}
\hline & Patient 1 & Patient 2 & \multicolumn{1}{c}{ Patient 3 } & \multicolumn{1}{c}{ Patient 4 } \\
\hline Relevant-use symptom* & cough & cough & nausea & nausea \\
Irrelevant-use symptom & sore muscles & earache & sore muscles & earache \\
Nonpredictive symptom $\dagger$ & numb fingers & skin rash & swollen tongue & inflamed knee \\
Treatment & galudane & galudane & veptendrine & veptendrine \\
\hline
\end{tabular}

Note-The cards given to the subjects contained just the three symptoms listed for each patient. ${ }^{*}$ Cough is a relevant-use symptom for subjects getting these materials, because whenever it occurs, the treatment to be given is galudane. Nausea always is treated with veptendrine. $†$ The nonpredictive symptoms were also presented with the other disease, buragamo. 
symptoms: Half the subjects received each set of cards (in which the particular relevant-use and irrelevant-use symptoms had been counterbalanced).

\section{Results and Discussion}

All 20 of the subjects learned both the diseases and treatments before the six-block limit. The diseases were learned in a mean of 3.1 blocks, and the treatments were learned in a mean of 2.5 blocks. The critical question was whether the four relevant-use symptoms (two for each disease) were responded to differently on tests than the four irrelevant-use symptoms that were also perfectly predictive of the diseases (but were not predictive of the treatment). Did the additional treatment task affect disease classification, even when the classification was learned to a high criterion first?

Single-symptom tests. If the treatment task affected classification, the relevant-use symptoms should have led to more accurate disease classifications than the irrelevant-use symptoms. They did. For classification accuracy, relevant-use symptoms led to the correct disease .90 of the time, and irrelevant-use symptoms .76 of the time $[t(19)=2.37, p<.05]$. Throughout this paper, a more sensitive additional measure will be used that combines the accuracy score with the confidence rating. For each test, the accuracy ( 1 for correct and 0 for incorrect) was multiplied by the confidence, and the average of this accuracy-confidence score was obtained. ${ }^{1}$ This accuracyconfidence score also showed an advantage for the relevant-use symptoms [5.2 vs. $4.3 ; t(19)=2.25, p<.05]$.

Double-symptom tests. The double-symptom tests consisted of eight critical conflict tests and eight filler tests. For the eight critical tests, a relevant-use symptom from one disease was paired with an irrelevant-use symptom for the other disease. The prediction, given that the relevant-use symptom was a better disease predictor, was that these tests would more often be classified by the disease of the relevant-use symptom. The results were consistent with that prediction for .64 of the responses $[t(19)=2.10, p<.05]$. The confidence scores were also used for these double-symptom tests by summing the confidences for the tests in which the subject's responses were consistent with the prediction in comparison with the tests in which the responses were inconsistent with the prediction. These sums are an indication of the relative confidence for and against the predictions. The sums were divided by eight so that they were roughly in the same range as the confidence rating. (Note that this procedure will generally lead to lower numbers than those for the single-symptom tests, because each of the eight confidence scores contributes to either the consistent or inconsistent sum.) The predictions that were consistent with performance determined by relevant-use symptoms had a higher confidence score than did the predictions that were inconsistent [ $3.3 \mathrm{vs.} 1.7 ; t(19)=2.58, p<.05$ ]

Experiment 1 provides evidence for a postclassification category use effect: The use of the category affected later classification judgments even when the learning of the use was not introduced until after classification learning. More specifically, even though the subjects first learned to classify patients into disease categories, the knowledge learned from the treatment judgments affected later disease classifications. The relevant-use symptoms and irrelevant-use symptoms were equally valid for disease classification, they were presented equally often, and they received the same feedback for the disease classification. Despite these similarities in classification learning, the relevant-use symptoms were classified more accurately than the irrelevant-use symptoms. Ross (1997) showed a category use effect in the interleaved learning paradigm with these common category learning materials, but the present Experiment 1 extended the result to a postclassification paradigm.

When people learn to make use of a category to make further predictions, the use can influence how classifications are made, even when the classification has been learned previously. This effect indicates that classification learning cannot be totally separated from category use, as many current theories assume. Rather, we need theories of categorization in which the classification learning can be affected by nonclassification use.

\section{EXPERIMENT 2}

Experiment 2 had two purposes: to extend the postclassification effect to another dependent measure and to begin to examine what might be necessary for getting such an effect. First, an extension to another dependent measure would both increase the generality of the finding and help us to understand what aspects of the task were important for the effect. Ross (1997) contrasted classification measures, which require symptom-todisease connections, with the possibility that the treatments might be making the relevant-use symptoms more accessible for other tasks as well. For example, in Ross (1997, Experiment 4), subjects learned the material as they had in the other experiments in that paper (interleaved), but then they were given a disease and were asked to generate symptoms that a person with the disease was likely to have. The relevant-use symptoms were generated more often than the irrelevant-use symptoms (.75 vs. .53) and were generated earlier. Thus, rather than make use of the symptom-to-disease connection from learning, in Experiment 2 I examined whether the relevantuse symptoms would also be more likely to be generated from the disease. This feature generation task is an unusual one for experimentally learned materials, but it does tap an important aspect of category knowledge (i.e., related to category validity measures) that is different from that used for classification.

Second, in Experiment 1, subjects learned to predict the appropriate treatment but also continued to make disease classification judgments on each trial. This procedure ensured that any effect of the treatment use on the 
disease classification would not occur because the classification had been forgotten during the learning of the use. In addition, this continued classification might be common in many cases in which people are learning a use for a category whose members they have previously learned to classify. A critical question for developing models of category use is to ask what is necessary during the learning of the use for there to be an effect on later classification. The remaining experiments addressed two parts of this question. One: Does the postclassification category use effect depend on having the category representation activated during the learning of the use? That question was the focus of Experiment 2. Two: if the category representation must be activated, is it necessary for the learning of use to be interleaved with additional explicit classification learning? That issue was the focus of Experiments 3, 4, and 5.

One suggestion in the introduction was that the postclassification category use effect depends on the category representation's being activated during the learning of the use. The idea is that this activated representation can be augmented by the learning of the use, and that the augmented representation is evaluated in making later classification decisions. For example, if one is learning that cough is predictive of galudane for terrigitis patients, the importance of cough in the terrigitis representation (i.e., its feature weight) is increased and may have more of an influence for later category-related judgments, such as classification. A different possibility is that the activation of the category representation during use learning is unnecessary - that what is important is that the relevant-use symptoms gain additional weighting during the learning of treatments. These weighted symptoms will then have much more influence in any later task (not unlike the Lawrence, 1949, idea that cues used in one discrimination may be more likely to be used in other discriminations). Thus, this alternative argues for a more general weighting of the relevant-use symptoms, not one tied only to the appropriate category representation. In Experiment 2, these views were contrasted by including a condition, called the separate condition, in which the use was learned without any reference to the categories. That is, following classification learning, subjects then learned to assign the appropriate treatment to each patient, but no mention was made of the diseases. Given the first view, this should lead to no category use effect (i.e., no difference in generation of relevant-use vs. irrelevant-use symptoms), whereas according to the second view, there should still be a difference.

\footnotetext{
Method

Subjects. The subjects were 40 University of Illinois undergraduates who participated for pay. The sessions lasted from 40 to $55 \mathrm{~min}$. Four additional subjects were replaced for not meeting the learning criteria described below.

Design and Materials. There was one between-subjects manipulation following the classification-only phase: Subjects either
}

learned the treatments with the interleaved disease classification and treatment prediction task as in Experiment 1 (the interleaved condition), or they learned the treatments without any reference to the disease classification (the separate condition). The design and study materials for the interleaved condition were exactly as in Experiment 1 . The only change in the separate condition was that during the treatment learning, the sheets the subjects saw that listed the possible treatments did not include the diseases. The test materials from Experiment 1 were not used, but were replaced by a generation task described below.

Procedure. The procedure for the disease classification learning phase was exactly as in Experiment 1 . However, if the subjects did not meet the learning criterion (responding correctly to at least 14 of 16 diseases on a block by Block 6), they were replaced. In the learning of treatments, the interleaved condition proceeded as in Experiment 1. For the separate condition, however, the subjects did not classify the patient into a disease category before deciding on the treatment. Rather, one of two sheets (containing either the buragamo treatments or the terrigitis treatments) was put in front of the subjects, and they were told that this patient should be treated by one of these two treatments. No mention was made of the disease. After learning both the disease classification and the treatments, the subjects were given each disease (the order was counterbalanced) and asked to list symptoms that a person with this disease would be likely to have. No mention was made of how many symptoms they should list.

\section{Results and Discussion}

In the interleaved condition, the learning procedure and materials were just as in Experiment 1, and the mean numbers of blocks needed to reach the learning criteria were 2.8 for the disease and 3.4 for the treatments. For the separate condition, the corresponding numbers were 2.6 and 2.6 .

The critical question was whether the relevant-use symptoms were more available for the disease than the irrelevant-use symptoms in the separate condition. The view that a connection is necessary between the use and classification predicted no difference between relevant-use and irrelevant-use symptoms, whereas the symptom weighting view predicted that relevant-use symptoms would continue to have an advantage in the separate condition. The relevant-use versus irrelevant-use difference was addressed by several measures, all of them showing an advantage for relevant-use symptoms in the interleaved condition, but not in the separate condition. The relevant-use versus irrelevantuse effect was larger for the interleaved condition, as predicted by the first view, for all the measures, though the difference was not always statistically significant.

The simplest measure was the proportion of (correct) relevant-use symptoms generated versus irrelevant-use symptoms. In the interleaved condition, the relevant-use symptoms were more likely to be generated than the irrelevant-use symptoms $[.80$ vs. .58 , respectively; $t(19)=$ $2.32, p<.05$. Note that these numbers are not very different from the .75 vs. .53 found in Ross, 1997 , when the classification and use were learned interleaved without prior classification learning]. In the separate condition, the corresponding mean proportions of .40 and .38 were much lower and did not significantly differ $[t(19)=$ 
$0.40]$. The .22 difference in the interleaved condition was not quite statistically significant from the .02 difference in the separate condition $[t(38)=1.69, p<.10]$.

A second measure took into account the order of the symptoms, as in Ross (1997, Experiment 3). Because no subject generated more than six symptoms for any disease, a simple scale was used in which 6 "points" were given for the first symptom generated, 5 for the second, and so forth. These data can be used to address two different questions: First, and foremost, how does the relevant-use versus irrelevant-use symptom difference compare across the two conditions? The number of points for all the relevant-use symptoms can be compared with those for the irrelevant-use symptoms to get a measure that may be more sensitive than the proportion of symptoms generated. In the interleaved condition, the corresponding averages (the sum of the number of points for the generated symptoms divided by the four symptoms of each type) were 4.0 and $2.5[t(19)=3.38, p<.01]$. The separate condition difference was small, 2.1 versus 1.7 , and not significant $[t(19)=1.18]$. The interleaved condition did show a greater difference than the separate condition $[t(38)=2.15, p<.05]$. Thus, the marginal effect with proportion of accurate classifications was statistically significant when the order of generation was taken into account. Note that this advantage occurred even though in the interleaved condition there was about twice as much practice in classifying the patients (because classification continued during the use learning).

The second question was whether the relevant-use symptoms would be generated earlier than the irrelevantuse symptoms, even taking into account the difference in the proportions of generation. To address this question, the number of points was divided by the number of generated symptoms (rather than by the total number of symptoms) to get an average number of points per generated symptom. Interestingly, the relevant-use symptoms showed an advantage in both conditions and of about the same magnitude: 5.0 versus 4.0 for the interleaved condition $[t(19)=2.81, p<.05]$, and 5.2 versus 4.1 for the separate condition $[t(19)=2.26, p<.05]$.

This overall pattern suggests that in the interleaved condition, the relevant-use symptoms were more likely both to be generated and to be generated earlier. In the separate condition, although the relevant-use symptoms were not generated more often, they were generated earlier than the irrelevant-use symptoms, indicating at least some influence of the use on the classification. A more detailed look at the data suggests that half of this effect was due to 2 subjects; however, even without these 2 subjects, there was a slight nonsignificant advantage for relevant-use symptoms in the order of generation.

This experiment added to our understanding of the postclassification category use effect in two ways: First, the effect was found not only with classification, but with another category-related measure, generation, in which the category-to-symptom information is crucial. Second, the effect was greatly reduced (to near zero for most measures) when the use learning did not activate the category representation. The nature of this connection between use learning and the category representation was explored further in Experiment 3.

\section{EXPERIMENT 3}

In this experiment, the goal was to examine the claim that the category use effect in this postclassification paradigm depended on the category representation's being activated during the learning of the category use. In Experiment 2 , the interleaved classification-use learning following classification learning led to a category use effect, whereas the separate learning of the use did not. One interpretation, which motivated the manipulation in Experiment 2, was that this difference occurred because the separate learning did not activate the category representation during the learning of the category use. A second, more restricted, interpretation was that the interleaved learning of classification and use is critical for obtaining the category use effect. By this interpretation, it is not simply the activation of the category representation during the learning of use that is critical, but rather that the learning of the use be tied in with the continued explicit learning of the classification. For example, perhaps subjects are looking to find a simple rule that will allow them to predict both the disease classification and the treatment use (e.g., "If the patient has a cough, then the disease is terrigitis and the treatment is galudane."). Even though they have already learned a good means of classification, interleaving the classification and use forces them to reconsider their current means of classification. The question of interest in Experiment 3 was whether the interleaving of classification and use would be necessary for obtaining the category use effect in this paradigm.

In Experiment 3, these two possible interpretations were contrasted as well as possible by including a condition very similar to the separate condition, except that the category representation was activated. More specifically, in one condition the subjects were told the correct disease category when they were given the patient's symptom set. This manipulation changed the explicit interleaved learning of classification and use to one in which the classification learning was greatly reduced. Should this condition (called given, because the disease classification was given to subjects) still lead to a postclassification category use effect, the continued explicit learning of the category during the use learning phase would not be necessary.

Rather than test this idea with the generation dependent measure, the classification dependent measure from Experiment 1 was used in Experiment 3. This change was made both because it is a more common measure of category representation than the feature generation task and also to replicate the main finding of Experiment 2, the lack of a category use effect in the separate condition, with a different dependent measure. 


\section{Method}

Subjects. The subjects were 46 University of Illinois undergraduates who participated for pay or course credit. The sessions lasted from 35 to $50 \mathrm{~min}$. Eleven additional subjects who did not meet the learning criteria were replaced.

Design and Materials. The design, study materials, and test materials were the same as in Experiment 1 . The only addition was a between-subjects manipulation for the learning of the treatments. About half of the subjects $(n=24)$ received no disease category information during treatment learning, as in the separate condition of Experiment 2. The other subjects $(n=22)$, in the given condition, were told the disease that each patient had before being asked to decide on the treatment. This between-subjects manipulation was also accompanied by a change in the sheets shown subjects from which they chose the two treatments. The separate condition sheets had "Possible Treatments" at the top, whereas the given condition sheets had "Possible Treatments for Terrigitis," or "Possible Treatments for Buragamo."

Procedure. The study procedure for the separate condition was exactly as in Experiment 2. For the given condition, the subjects were told during the treatment learning phase what disease each patient had before being asked to decide on the treatment. The test for all the subjects consisted of the single-symptom and double-symptom tests as in Experiment 1.

\section{Results and Discussion}

Learning. In the given condition, learning was faster than in the earlier experiments, with mean numbers of blocks of 2.3 for the disease classification and 2.1 for the treatments. In the separate condition, the learning was somewhat slower than usual, with means of 3.7 and 3.4. Note that the classification learning phase was the same in both conditions. Of the 11 subjects replaced, 7 did not meet the classification learning criterion, and the other 4 did not meet the treatment learning criterion for the separate condition.

Single-symptom tests. If the treatment task was affecting classification even when the classification was given, the relevant-use symptoms should again lead to more accurate disease classifications than should the irrelevant-use symptoms in the given condition. The combined accuracy-confidence score did show a difference, with means of 5.2 versus $4.3[t(21)=2.51, p<.05]$. The accuracy score did not show any difference, with proportions of .85 for both conditions. This result is surprising, but may be partly due to the high performance and the problem of ceiling effects (which is one reason why the accuracy-confidence score was introduced in Ross, 1997, and used in Experiment 1 as well). In the relevant-use condition, 14 of the 22 subjects were correct on all four items ( 10 of 22 subjects for the irrelevantuse symptoms). ${ }^{2}$

The separate condition showed no advantage for the relevant-use symptoms, with the irrelevant-use symptoms classified nonsignificantly more accurately $[.67 \mathrm{vs}$. $.56 ; t(23)=1.55]$. The combined accuracy-confidence score did not show any difference either [ $2.6 \mathrm{vs}$. 2.5 ; $t(23)=0.03]$.

In the comparison of the two conditions, the given condition did not show a significantly greater effect than did the separate condition, though it was close for the combined measure $[t(44)=1.12$ for accuracy, and $t(44)=$ $1.87, p<.07$, for the accuracy-confidence measure].

Double-symptom tests. The double-symptom tests of interest paired a relevant-use symptom for one disease with an irrelevant-use symptom for the other disease. The prediction was that subjects would choose the disease for the relevant-use symptom. They did .70 of the time in the given condition $[t(21)=4.34, p<.01]$. This effect was particularly strong, occurring for 17 of 20 subjects (with two ties). The separate condition showed no difference, $.52[t(23)=0.43]$. The given condition proportion was statistically greater than that for the separate condition $[t(44)=2.66, p<.05]$. The same pattern of results occurred when the confidence was taken into account, with a significant difference for the given condition [ 3.2 vs. $1.3 ; t(21)=3.78, p<.01]$; no difference for the separate condition $[2.2$ vs. $1.9, t(23)=.68]$; and a significantly larger difference in the given condition than in the separate condition $[t(44)=2.36, p<.05]$.

The results of Experiment 3 suggest that the interleaved learning of classification and use was not necessary for obtaining a postclassification category use effect. The main result is that even when the disease classification was provided with the patient information (in the given condition), the relevant-use symptoms were viewed as better predictors of the disease category than were the irrelevant-use symptoms in both the combined singlesymptom test and the double-symptom test measures. In addition, the lack of an effect with the separate condition learning procedure was replicated from Experiment 2, but now with a classification test. Although the interaction between the two conditions was significant only in the double-symptom test, the experiment does show that interleaving is not necessary in order for category use to affect classification, even when the use is learned after the classification.

\section{EXPERIMENT 4}

Experiment 3 showed that providing the classification during use learning was sufficient to lead to a postclassification category use effect, but this effect did not occur for the accuracy proportions of single-symptom classification tests. From a further examination of the data, it appeared that this lack of effect might have been due to ceiling effects for many subjects, combined with the particularly poor performance of a few subjects on the relevant-use symptoms. Experiment 4 was a replication of the given condition of Experiment 3, to check whether this lack of effect on single test accuracy was likely to be real or attributable to statistical fluctuation.

\section{Method}

Subjects. The subjects were 30 University of Illinois undergraduates who participated as part of a class assignment or for pay. The sessions lasted from 30 to $45 \mathrm{~min}$. Fourteen additional subjects were 
excluded for failure to meet the learning criteria for disease category and treatment use--though note that almost all of them (11) failed to meet the disease category learning criterion. Omitting them should not help lead to a postclassification category use effect.

Design, Materials, and Procedure. The study and test materials were exactly as in Experiment 3. The procedure for all subjects was as in the given condition of Experiment 3.

\section{Results and Discussion}

Learning. The learning for these given condition subjects required means of 3.4 blocks for disease classification and 3.0 blocks for the treatments.

Single-symptom tests. If the treatment task was affecting classification even when the classification was given, the relevant-use symptoms should lead to more accurate disease classifications than should the irrelevantuse symptoms in the given condition. In Experiment 3, there was no difference for the accuracy proportions, with .85 for both, but there was a difference in the combined accuracy-confidence score. In Experiment 4, both measures showed a significant difference. For accuracy, the relevant-use symptoms' proportion of .86 was greater than the .74 for irrelevant-use $[t(29)=2.63, p<.05]$. For the combined accuracy-confidence score, the corresponding means were 4.9 and $3.7[t(29)=4.06, p<.01]$. Thus, it appeared that the given condition could lead to a significant accuracy effect on single classification tests.

Although it involved a cross-experiment comparison, the given condition of Experiment 4 did show a greater relevant-use versus irrelevant-use effect than did the separate condition in Experiment 3, both for accuracy $[t(52)=2.68, p<.01]$ and for the combined accuracyconfidence score $[t(52)=2.56, p<.05]$.

Double-symptom tests. The double-symptom tests of interest paired a relevant-use symptom from one disease with an irrelevant-use symptom from the other disease. As before, subjects chose the disease for the relevant-use symptom more often, with a proportion of $.65[t(29)=$ $3.36, p<.01]$. The given condition proportion was marginally greater than that for the separate condition in Experiment $3[t(52)=1.94, p<.06]$. The confidence scores led to the same pattern with a relevant-use versus irrelevant-use difference of 3.3 versus $1.6[t(30)=3.63, p$ $<.01]$, a difference significantly greater than that for the separate condition in Experiment $3[t(52)=2.09, p<.05]$.

The results of this experiment clarify the findings of Experiment 3 by demonstrating an effect even for the single-symptom accuracy measure. Thus, Experiments 3 and 4 showed that providing the classification at the time of treatment learning is sufficient for obtaining a postclassification category use effect.

\section{EXPERIMENT 5}

Experiment 5 replicated the given condition with the generation measure used in Experiment 2 . The primary goal of this experiment was to extend the effect of the given condition to a different category-related measure.
Would providing the classification during the use learning lead to an effect on the generation of symptoms for each disease? In addition, this experiment provided a completeness to the manipulations. The classification measure had been examined with the interleaved (classification and use learning) condition (Experiment 1), the separate condition (Experiment 3 ), and the given condition (Experiments 3 and 4 ). The generation measure had been examined with the interleaved condition (Experiment 2) and the separate condition (Experiment 2). Although there was no clear way to compare the size of the effects across the two measures, the qualitative patterns of effects and noneffects with these different measures might be important in helping to understand the postclassification category use effect.

\section{Method}

Subjects. The subjects were 22 University of Illinois undergraduates who participated as part of a class assignment or for pay. The sessions lasted from 30 to $50 \mathrm{~min}$. Because so many subjects did not meet the learning criterion in earlier experiments, we included all subjects here who had at least 9 of 16 correct responses by the final block of each learning. This only excluded 1 subject, but in fact all but 3 of the 22 final subjects met the previous criteria of 14 of 16 correct responses on the final block.

Design, Materials, and Procedure. The study materials, test materials, and learning procedures were exactly as in Experiment 4. The test procedure was the generation paradigm used in Experiment 2 . All subjects were in the given condition.

\section{Results and Discussion}

The mean numbers of blocks needed to reach the learning criteria were 3.6 for the disease and 3.3 for the treatments.

As in Experiment 2, a few measures compared relevantuse symptoms with irrelevant-use symptoms, and all showed an advantage for the generation of relevant-use symptoms. First, the proportion of (correct) relevant-use symptoms generated, .71 , was much greater than the proportion of irrelevant-use symptoms generated, .39 $[t(21)=5.91, p<.01]$. Second, taking into account the order of the symptoms (with 6 "points" given for the first symptom generated, 5 for the next, etc.), the average numbers of points for the relevant-use and irrelevant-use symptoms were 3.6 and $1.8[t(21)=6.10, p<.01]$. Third, the relevant-use symptoms were generated earlier than the irrelevant-use symptoms, even if we take into account the difference in the proportions of generation, with an average number of points per generated symptom of 5.1 versus $3.9[t(21)=2.59, p<.05]$.

No separate condition was tested in Experiment 5, because the main goal was to see whether the given condition manipulation led to an effect for the generation measure. However, if one uses the separate condition of Experiment 2 as a control, the given condition led to a greater postclassification category use effect for proportion generated $[t(40)=2.79, p<.01]$ and for points generated $[t(40)=3.41, p<.01]$, but not for the mean points of generated symptoms $[t(40)=.15]$. 
Table 2A

Results From Experiment 1

\begin{tabular}{cccccccc}
\hline & & \multicolumn{4}{c}{ Classification Tests } \\
\cline { 3 - 6 } $\begin{array}{c}\text { Postclassification } \\
\text { Condition }\end{array}$ & Symptoms & $\begin{array}{c}\text { Single } \\
\text { Proportion } \\
\text { Accuracy }\end{array}$ & $\begin{array}{c}\text { Accuracy- } \\
\text { Confidence }\end{array}$ & $\begin{array}{c}\text { Proportion } \\
\text { Consistent With } \\
\text { Relevant Use }\end{array}$ & Accuracy- \\
Confidence
\end{tabular}

Table 2B

Results From Experiment 2

\begin{tabular}{ccccc}
\hline & & \multicolumn{3}{c}{ Generation Tests } \\
\cline { 3 - 5 } $\begin{array}{c}\text { Postclassification } \\
\text { Condition }\end{array}$ & Symptoms & $\begin{array}{c}\text { Proportion } \\
\text { Generated }\end{array}$ & $\begin{array}{c}\text { Average } \\
\text { Points }\end{array}$ & $\begin{array}{c}\text { Conditionalized } \\
\text { Points }\end{array}$ \\
\hline Interleaved & Relevant use & .80 & 4.0 & 5.0 \\
& Irrelevant use & .58 & 2.5 & 4.0 \\
Separate & Relevant use & .40 & 2.1 & 5.2 \\
& Irrelevant use & .38 & 1.7 & 4.1 \\
\hline
\end{tabular}

Table 2C

Results From Experiment 3

\begin{tabular}{|c|c|c|c|c|c|}
\hline \multirow[b]{3}{*}{$\begin{array}{l}\text { Postclassification } \\
\text { Condition }\end{array}$} & \multirow[b]{3}{*}{ Symptoms } & \multicolumn{4}{|c|}{ Classification Tests } \\
\hline & & \multicolumn{2}{|c|}{ Single } & \multicolumn{2}{|c|}{ Double } \\
\hline & & $\begin{array}{c}\text { Proportion } \\
\text { Accuracy }\end{array}$ & $\begin{array}{l}\text { Accuracy- } \\
\text { Confidence }\end{array}$ & $\begin{array}{c}\text { Proportion } \\
\text { Consistent With } \\
\text { Relevant Use }\end{array}$ & $\begin{array}{l}\text { Accuracy- } \\
\text { Confidence }\end{array}$ \\
\hline Given & & & & .70 & \\
\hline \multirow{5}{*}{ Separate } & Relevant use & .85 & 5.2 & \multirow[b]{3}{*}{.52} & \\
\hline & Irrelevant use & .85 & 4.3 & & \\
\hline & & & & & \\
\hline & Relevant use & .56 & 2.5 & & \\
\hline & Irrelevant use & .67 & 2.6 & & \\
\hline
\end{tabular}

Table 2D

Results From Experiment 4

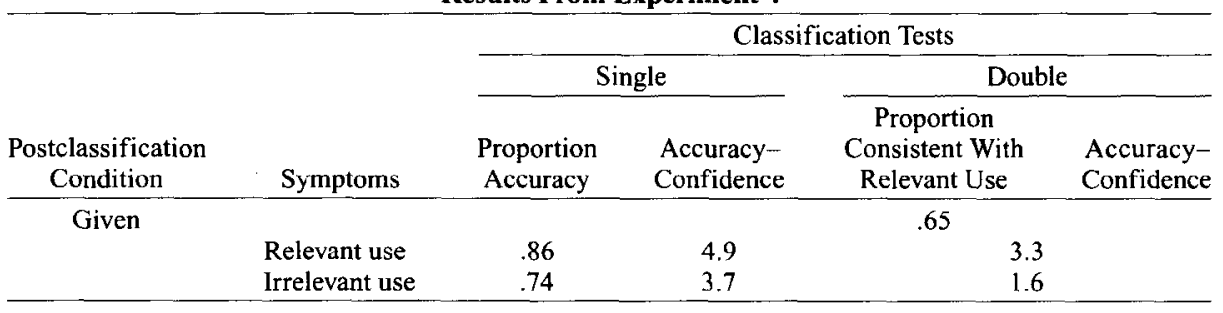

Table 2E

Results From Experiment 5

\begin{tabular}{ccccc}
\hline \multirow{2}{*}{$\begin{array}{c}\text { Postclassification } \\
\text { Condition }\end{array}$} & Symptoms & $\begin{array}{c}\text { Proportion } \\
\text { Generated }\end{array}$ & $\begin{array}{c}\text { Generation Tests } \\
\text { Pverage }\end{array}$ & $\begin{array}{c}\text { Conditionalized } \\
\text { Points }\end{array}$ \\
\hline Given & Relevant use & .71 & 3.6 & 5.1 \\
& Irrelevant use & .39 & 1.8 & 3.9 \\
\hline
\end{tabular}

This overall pattern suggests that in the given condition, the relevant-use symptoms were more likely both to be generated and to be generated earlier.

\section{GENERAL DISCUSSION}

Category learning consists of both learning to classify and learning to use the category. The present experi- ments show that the learning of the category use can af fect the category representation, including knowledge used to classify later instances, even if the use is learned after the classification has been learned. The implications of the category use effect for theories of classification have been considered in earlier papers (Ross, 1996a, 1997), so here the focus is on the generality of the findings and an account of the postclassification effects. 
First, a short summary of the results will be provided to make more clear what the conditions for obtaining such an effect are.

\section{Summary of the Results}

The results of these experiments extend our understanding of how category use can affect later classification. The earlier work showed that category use could affect the category representation when the learning for each of the two tasks was interleaved. It is possible that such effects occur only when the tasks are learned in this way, because under such circumstances learners might try to find features that provide accurate responses in both tasks, so it is important to consider other learning paradigms. In the experiments reported here, the use of the categories was not introduced until after subjects had learned to classify the items, yet the use still affected later classifications. In addition to this demonstration of the postclassification effect, conditions were considered under which the effect might occur, and the effects on another category-related measure besides classification, feature generation, were examined. Because of the many different results over the five experiments, Tables $2 \mathrm{~A}-2 \mathrm{E}$ contain a summary of the findings.

A common disease classification procedure was used in all of the experiments, but following the learning of disease, subjects had to learn to predict which treatment would be given to each patient. Experiment 1 demonstrated the basic postclassification category use effect on classification performance. In the remaining experiments, some conditions were explored under which the effect might occur and the generality of the effect was broadened. In Experiment 2, this work was extended to show both that the effect occurred with a different dependent measure (generation of features from the category) and that the effect did not occur if the classification was not required when one was learning the use. Experiment 3 showed that it was not necessary to have interleaved learning of the classification and use when one was learning the use--the effect occurred even when the classification was given before the category was used. In addition, Experiment 3 replicated the noneffect in the separate condition with the classification measure. Experiment 4 replicated the given condition of Experiment 3 and showed an effect even on the single-symptom accuracy. Finally, Experiment 5 showed that giving the classification affected later feature generation as well.

Thus, the pattern of results is fairly consistent: Across both dependent measures (classification and feature generation), one finds a postclassification category use effect for the interleaved and given conditions, but no such effect for the separate condition.

Although this summary statement captures the major trends in the studies, three inconsistencies in the results should be mentioned. First, in the given condition of Experiment 3 , there was no effect on the accuracy measure for the single-symptom test, although there was an effect for the combined accuracy-confidence score. The lack of difference may have been due to ceiling effects, and the replication in Experiment 4 did yield a significant effect on accuracy. Second, although the separate condition usually showed little effect of the use on later tests, Experiment 2 did find that the relevant-use symptoms were generated earlier than the irrelevant-use symptoms. The exact reason for this effect is unclear, but it is possible that the manipulation did not totally succeed in avoiding activation of the category representation during the learning of the use (Ross, 1999, examines when this might occur). Third, there were large differences across experiments in the proportion of subjects who failed to meet the learning criterion, even for the classification learning, which was the same across all the conditions. I have no explanation for this variation except to point out that the experiments were run successively, so that subjects were tested at different times of the year. It is most important to note, however, that a majority of the subjects excluded were done so on the basis of the classification (disease) learning criterion, at which point the manipulation of symptom predictiveness (relevant use vs. irrelevant use) and the between-subjects manipulations (e.g., interleaved vs. separate) had not yet been introduced. Thus, this unexplained variation is unlikely to have inadvertently led to any of the effects seen in these experiments.

\section{Generality of Category Use Effect \\ Learning paradigms. A primary goal of this research} was to extend the category use effect findings of Ross (1997) to a different learning situation. Ross (1997) showed that if the classification and use were learned in an interleaved paradigm, the use learning affected later classifications. The experiments reported here showed the same effect with a postclassification paradigmwhen the use was learned after the classification had been learned, it still had an effect on later classifications.

The postclassification paradigm was chosen for two reasons. First, like the interleaved learning situation, the postclassification learning paradigm is common in realworld learning. In some cases, we learn about the types of items in a domain and only later learn a use of the category. For example, many biological domains, such as dogs, may be learned in this way - we initially learn to classify some animals as dogs, but later we can use the category to make predictions or inferences about particular dogs. In other cases, we may learn a new use for an already learned classification, as may happen with additional expertise in an area. Second, the postclassification paradigm was chosen because it provides an extreme difference from the interleaved paradigm. The findings now show an effect of use on classification if the use is learned with the classification and if the use is learned after the classification. Thus, it seems likely that one might also get such an effect in the many intermediate cases in which the classification is partially learned before a category use is introduced. Although there may well be additional ways in which categories 
are learned, the goal was to show that the category use effect occurred in two very different learning paradigms.

Category uses. In addition to showing some generality across learning paradigms, the research has also been concerned with showing generality across category uses. In particular, I have addressed two very different types of category uses: feature prediction and problem solving. With the interleaved learning paradigm, both types of category uses affect later classification (Ross, 1997). The results presented here show that the feature prediction affects later classification with the postclassification paradigm as well. Ross (1999), also with a postclassification paradigm, found that the problem-solving use affected later classifications. Thus, it appears that both types of category uses show effects on classification.

\section{Accounting for the Postclassification Category Use Effect}

The category use effect goes beyond the situations investigated by classification models to ask how additional nonclassification learning might affect the classification. Because of this, the results do not falsify these views, but rather suggest ways in which they need to be extended. Ross (1997) examined how three important classification models, prototype models (e.g., Reed, 1972; Rosch, 1978), ALCOVE (Kruschke, 1992), and MINERVA2 (Hintzman, 1986, 1988), might account for the category use effect in the interleaved learning paradigm. To summarize that discussion, the first two models do not have an obvious means for a nonclassification use to affect the classification judgment. It is possible that ALCOVE could be modified to include the uses that depend on the category. For example, the treatments could be connected to both the symptoms and diseases so that the prediction of the treatments (by the relevant-use symptoms) would add extra weight to the category as well. (The prototype modifications are less clear since there is no agreed-upon learning mechanism.) MINERVA2, because it is meant to be a general memory model rather than only a classification model, is able to incorporate nonclassification uses for classification judgments. The main modification needed for this model is a way of differentially weighting features within its representation, which seems well motivated by other results as well (see Ross, 1997, pp. 261263 , for a more complete discussion).

For the postclassification paradigm, the accounting of the effects is fairly similar to the interleaved paradigm case. For the classification learning, all models are able to predict that some or all of the relevant-use and irrelevantuse symptoms are going to be learned as predictive of the disease category. The interesting part of the account is what happens when irrelevant-use symptoms have been learned as predictive of the category but during the treatment learning the relevant-use symptoms are predictive. The result is that the relevant-use symptoms are viewed as more central to the category representation (as seen in classification and generation). Two general pos- sibilities may be distinguished. First, the treatment learning leads to a general weighting of the relevant-use symptoms (such that they receive more attention or weighting across a variety of tasks). The lack of a category use effect in the separate condition argues against such a general weighting (or at least such a general weighting being a determinant of the category use effect). Second, the treatment learning leads to a weighting within the category representation of the disease. As argued here, one likely possibility for such an effect is that the category representation is activated during the learning of use (either because the subjects must classify the instance or because it is provided). Such an interpretation is bolstered by the results of Ross (1999) in which the postclassification category use effect occurred even in the separate condition when subjects were likely to be incidentally activating the category representation (because of additional processing or extended learning).

The postclassification category use effect shown in the experiments in this article could have been due to a simple differential weighting of relevant-use symptoms. The main challenge to current classification theories is to understand how later learning affects the classification judgments, and why this effect did not occur in the separate condition. Note, however, that the problem-solving findings (Ross, 1999) are not explainable in terms of a simple weighting, and it is not clear how best to incorporate these more complex use effects into current theories.

\section{Conclusions}

Often we learn to classify items in a domain and then gain additional knowledge about these learned concepts, such as how to make use of them to accomplish some goal. Is the original classification affected by the later learning? The present research suggests that it is, and provides some preliminary results on the conditions under which the effect occurs. In particular, it appears that during the additional learning, the original category representation must be activated so that it can be changed. Thus, this work further extends category learning beyond classification learning to address how it might be affected by additional learning.

\section{REFERENCES}

ANDERSON, J. R. (1991). The adaptive nature of human categorization. Psychological Review, 98, 409-429.

Barsalou, L. W. (1983). Ad hoc categories. Memory \& Cognition, 11, 211-227.

BARSALOU, L. W. (1985). Ideals, central tendency, and frequency of instantiation as determinants of graded structure in categories. Journal of Experimental Psychology: Learning, Memory, \& Cognition, 11, 629-654

BARSALOU, L. W. (1991). Deriving categories to achieve goals. In G. H. Bower (Ed.), The psychology of learning and motivation (Vol. 27, pp. 1-64). New York: Academic Press.

BILLMAN, D., \& Herr, E. (1988). Observational learning from internal feedback: A simulation of an adaptive learning method. Cognitive Science, 12, 587-625.

BILlMaN, D., \& KNUTSON, J. (1996). Unsupervised concept learning 
and value systematicity. Journal of Experimental Psychology: Learning, Memory, \& Cognition, 22, 458-475.

BOSTER, J. S., \& JOHNSON, J. C. (1989). Form or function: A comparison of expert and novice judgments of similarity among fish. American Anthropologist, 91, 866-889.

ChI, M. T. H., Feltovich, P. J., \& Glaser, R. (1981). Categorization and representation of physics problems by experts and novices. $\mathrm{Cog}$ nitive Science, 5, 121-152.

Gelman, S. A., \& Markman, E. M. (1986). Categories and induction in children. Cognition, 23, 183-208.

Gibson, E. J. (1969). Principles of perceptual learning and development. New York: Appleton-Century-Crofts.

Hert, E. (1992). Categorization using chains of examples. Cognitive Psychology, 24, 341-380.

Hintzman, D. L. (1986). "Schema abstraction" in a multiple-trace memory model. Psychological Review, 93, 411-428.

Hintzman, D. L. (1988). Judgements of frequency and recognition memory in a multiple-trace memory model. Psychological Review, 95, 528-551.

Johnson, K. E., \& MERvis, C. B. (1997). Effects of varying levels of expertise on the basic level of categorization. Journal of Experimental Psychology: General, 126, 248-277.

KRUSCHKE, J. K. (1992). ALCOVE: An exemplar-based connectionist model of category learning. Psychological Review, 99, 22-44.

LAMBERTS, K. (1994). Flexible tuning of similarity in exemplar-based categorization. Journal of Experimental Psychology: Learning, Memory, \& Cognition, 20, 1003-1021.

LASSALINE, M. E., \& MURPHY, G. L. (1996). Induction and category coherence. Psychonomic Bulletin \& Review, 3, 95-99.

LAWRENCE, D. H. (1949). Acquired distinctiveness of cues: I. Transfer between discriminations on the basis of familiarity with the stimulus. Journal of Experimental Psychology, 39, 770-784.

Lopez, A., Atran, S., Coley, J. D., Medin, D. L., \& Smith, E. E. (1997). The tree of life: Universal and cultural features of folkbiological taxonomies and inductions. Cognitive Psychology, 32, 251295.

MALT, B. C. (1995). Category coherence in cross-cultural perspective. Cognitive Psychology, 29, 85-148.

Malt, B. C., Ross, B. H., \& MurPhy, G. L. (1995). Predicting features for members of natural categories when categorization is uncertain. Journal of Experimental Psychology: Learning, Memory, \& Cognition, 21, 646-661.

Markman, A. B., Yamauchi, T., \& Makin, V. S. (1997). The creation of new concepts: A multifaceted approach to category learning. In T. B. Ward, S. M. Smith, \& J. Vaid (Eds.), Creative thought: An investigation of conceptual structures and processes (pp. 179-208) Washington, DC: American Psychological Association.

Markman, E. M. (1989). Categorization and naming in children: Problems of induction. Cambridge, MA: MIT Press.

Medin, D. L., \& Edelson, S. (1988). Problem structure and the use of base rate information from experience. Journal of Experimental Psychology: General, 117, 68-85.

Medin, D. L., Lynch, E. B., Coley, J. D., \& Atran, S. (1997). Categorization and reasoning among tree experts: Do all roads lead to Rome? Cognitive Psychology, 32, 49-96.

Medin, D. L., \& Schaffer, M. M. (1978). Context theory of classification learning. Psychological Review, 85, 207-238.

Medin, D. L., \& Smith, E. E. (1984). Concepts and concept formation. Annual Review of Psychology, 35, 113-138.

MurPhy, G. L. (1993). A rational theory of concepts. In G. V. Nakamura, R. M. Taraban, \& D. L. Medin (Eds.), The psychology of learning and motivation: Vol. 29. Categorization by humans and machines (pp. 327-359). New York: Academic Press.

MURPhY, G. L., \& Ross, B. H. (1994). Predictions from uncertain categorizations. Cognitive Psychology, 27, 148-193.

NosorsKY, R. (1988). Similarity, frequency, and category representations. Journal of Experimental Psychology: Learning, Memory, \& Cognition, 14, 54-65.
Osherson, D. N., Smith, E. E., Wilkie, O., Lopez, A., \& Shafir, E (1990). Category-based induction. Psychological Review, 97, 185200 .

ReED, S. K. (1972). Pattern recognition and categorization. Cognitive Psychology, 3, 382-407.

Rosch, E. (1978). Principles of categorization. In E. Rosch \& B. Lloyd (Eds.), Cognition and categorization (pp. 27-48). Hillsdale, NJ: Erlbaum.

Ross, B. H. (1996a). Category learning as problem solving. In D. L. Medin (Ed.), The psychology of learning and motivation, (Vol. 35, pp. 165-192). San Diego: Academic Press.

Ross, B. H. (1996b). Category representations and the effects of interacting with instances. Journal of Experimental Psychology: Learning, Memory, \& Cognition, 22, 1249-1265.

Ross, B. H. (1997). The use of categories affects classification. Journal of Memory \& Language, 37, 240-267.

Ross, B. H. (1999). Postclassification category use: The effects of learning to use categories after learning to classify. Journal of Experimental Psychology: Learning, Memory, \& Cognition, 25, 743-757.

Ross, B. H., \& MurPHy, G. L. (1996). Category-based predictions: The influence of uncertainty and feature associations. Journal of Experimental Psychology: Learning, Memory, \& Cognition, 22, 736-753.

Ross, B. H., \& Spalding, T. L. (1994). Concepts and categories. In R. Sternberg (Ed.), Handbook of perception and cognition: Vol. 12. Thinking and problem solving (pp. 119-148). San Diego: Academic Press.

Schyns, P. G., Goldstone, R. L., \& Thibaut, J.-P. (1998). The development of features in object concepts. Behavioral \& Brain Sciences, 21, 1-54.

SchyNs, P. G., \& RoDET, L. (1997). Categorization creates functional features. Journal of Experimental Psychology: Learning, Memory, \& Cognition, 23, 681-696.

Smith, E. E., \& Medin, D. L. (1981). Categories and concepts. Cambridge, MA: Harvard University Press.

TANAKA, J. W., \& TAYLOR, M. E. (1991). Categorization and expertise: Is the basic level in the eye of the beholder? Cognitive Psychology, 23, 457-482.

WARD, T. B., \& BECKER, A. H. (1992). Learning categories with and without trying: Does it make a difference? In B. Burns (Ed.), Percepts, concepts and categories (pp. 451-491). Amsterdam: Elsevier.

WAXMaN, S. R., LYNCH, E. B., CASEY, K. L., \& BAER, L. (1997). Setters and samoyeds: The emergence of subordinate level categories as a basis for inductive inference in preschool-age children. Developmental Psychology, 33, 1074-1090.

Yamauchi, T., \& Markman, A. B. (1998). Category-learning by inference and classification. Journal of Memory \& Language, 39, 124148.

\section{NOTES}

1. The accuracy-confidence measure was used as the primary score for single-symptom tests to provide a more sensitive measure, because the classification accuracy proportion is based on only four observations per condition for each subject.

2. Of the subjects who showed any difference between the two types of symptoms, there were slightly more subjects with higher performance on the relevant-use symptoms ( 8 to 5 ). However, of the 5 subjects who accurately classified fewer than three of four symptoms of either type, 4 of the subjects did so for relevant-use symptoms. It is always hard to tell exactly what happened in cases such as these, but it does appear that what may have been a small advantage for the relevant-use symptoms was overwhelmed by the very poor performance of 4 subjects on those symptoms.

(Manuscript received July 13, 1998; revision accepted for publication January 14,1999 .) 